\title{
Optimización de la comunicación hospitalaria en redes sociales: Análisis y estrategias de engagement
}

\author{
Toni GonzÁlez PACANOWSKI \\ Universidad de Alicante \\ toni.gonzalez@ua.es
}

https://orcid.org/0000-0003-3005-2218

\author{
Pablo Medina Aguerrebere \\ Canadian University Dubai \\ pablo.medina@cud.ac.ae
}

https://orcid.org/0000-0002-4463-4721

\section{Eva Medina}

Universidad de Alicante

eva.medina25818@gmail.com

https://orcid.org/0000-0001-6276-4127

\author{
Paula López Molina \\ Universidad de Alicante \\ plm43@alu.ua.es
}

https://orcid.org/0000-0002-2560-9053

\section{Optimization of hospital communication in social networks. Analysis and engagement strategies}

\section{RESUMEN ABSTRACT}

La comunicación hospitalaria tiende cada vez más a maximizar los beneficios de la gestión de redes sociales y su engagement. Para lograr este objetivo, es clave el estudio analítico. Con el objetivo de establecer recomendaciones centradas en optimizar la estrategia de contenidos en las redes sociales de hospitales e identificar elementos de mejora, se presentan resultados obtenidos con la ayuda de diversas herramientas analíticas aplicadas a una muestra de centros hospitalarios de Alicante (España). Con base en el registro actual de la base de datos ObservaTICs, son descritos los perfiles de Facebook y Twitter, utilizando ratios para la medición del engagement y a través de la revisión del tráfico de sus páginas web. En conclusión, se observa que los centros, si bien pueden mostrar diferencias en cuanto a su actividad y calidad de contenido, requieren una planificación y control de las redes sociales para comunicarse con sus públicos, sus stakeholders y facilitar información útil sobre asuntos de salud que repercute en la reputación de la propia institución y especialmente en la salud de los mismos pacientes. En el futuro es probable que se incremente este tipo de comunicación mediante contenidos enfocados a reforzar los vínculos paciente-médico, así como paciente-hospital.

PALABRAS CLAVE

Comunicación; Marca; Salud; Redes sociales; Engagement.
Hospital communication tends more and more to maximize the benefits of social media management and engagement. To achieve this goal, analytical study is key. With the aim of establishing recommendations focused on optimizing the content strategy in hospital social networks and identifying elements for improvement, results obtained with the help of various analytical tools applied to a sample of hospital centers in Alicante (Spain) are presented. Based on the current registry of the ObservaTICs database, the Facebook and Twitter profiles are described, using ratios to measure engagement and through the traffic review of their web pages. In conclusion, it is observed that the centers, although they may show differences in terms of their activity and quality of content, require planning and control of social networks to communicate with their audiences, their stakeholders and provide useful information on health matters that It has repercussions on the reputation of the institution itself and especially on the health of the patients themselves. In the future, this type of communication is likely to increase through content focused on reinforcing patient-doctor links, as well as patient-hospital.

\section{KEYWORDS}

Communication; Brand; Health; Social media; Engagement. 


\section{Introducción}

\subsection{Escenario}

Con las nuevas exigencias informativas de los medios en contenidos de salud (Becerra Muñoz, et al., 2015), los nuevos públicos emergentes online del sector sanitario y ante el aumento de la competencia comunicativa entre hospitales de gestión pública y privada, se configura un escenario donde se hace cada vez más evidente que la reputación de un hospital está influenciada por su presencia en las redes sociales (Triemstra, Poeppelman y Arora, 2018).

En el caso español, un 50\% de los hospitales españoles utilizan Facebook, Twitter o YouTube como canales de comunicación. El resto de medios sociales no son utilizados por más de un tercio del sector (Costa, Túñez y Videla, 2016). En el ámbito de países como Estados Unidos la gran mayoría de los hospitales utilizan de manera abrumadora estas tres plataformas de redes sociales mencionadas (Smith, 2017).

Los hospitales públicos en España son más activos en Twitter, mientras que los hospitales privados muestran un mejor desempeño en Facebook y YouTube (Martínez-Millana et al., 2017). Por lo que se sugiere que los hospitales, tanto públicos como privados, deberían dedicar más esfuerzo y ser más conscientes de las redes sociales, con una estrategia clara sobre cómo pueden fomentar nuevas relaciones con pacientes y ciudadanos. De hecho, existen bajos índices de interacción mediante Twitter entre públicos de entornos con alto desarrollo sanitario. Dentro de los públicos target de un centro hospitalario es crucial el de los pacientes crónicos con las RRSS, ya que les permite compartir información con otros usuarios y establecer lazos con personas de su misma condición. Se ha observado que los pacientes una vez han interactuado con los perfiles institucionales de los hospitales incrementan su interés hacia ellos en gran medida (Rando, Paniagua y De las Heras, 2016). Por tanto, es clave que el hospital tenga presencia en redes sociales dirigidas y planificadas, que genere una imagen de marca y que se comunique con sus principales stakeholders (Kumar, Jacob y Thota, 2014). Resulta necesario que la marca del hospital se convierta en el elemento principal de cohesión de todas las partes integrantes de este (Naveen, Anil y Smruthi, 2014).

También parece evidente que las medidas agregadas de satisfacción del paciente en las redes sociales se correlacionan con las medidas de calidad hospitalaria aceptadas tradicionalmente (Glover et al., 2015). También se ha descrito el valor emocional mediante mensajes testimoniales como elemento decisivo en la comunicación hospitalaria con sus públicos en redes sociales (López, Valedarrama y Di, 2019).

Uno de los principales públicos objetivos que tiene un centro hospitalario son sus pacientes. La figura del usuario o paciente del centro es vital para la construcción de una imagen de marca. Es, además, un colectivo público informado, activo y exigente. Desde hace tiempo es un líder de opinión capaz de conseguir atención y de influir en la imagen de los centros hospitalarios. Los hospitales por tanto tienen la obligación de comunicar a dichos pacientes y a sus demás stakeholders información de calidad por parte de profesionales de la salud. En España

el $25,7 \%$ consulta en Internet antes de acudir a la consulta médica (ONTSI, 2016). No obstante, se considera que las redes sociales en dicho estudio no eran el medio mayoritario para la búsqueda de temas de salud y los ciudadanos tenían escasa confianza en él. Los usos que más destacan en RRSS, según dicho informe, son la búsqueda general de información sobre la salud y conocimientos sobre el cuidado personal y estado de salud.

Además, de todos los sectores sanitarios, el hospitalario parece ser el menos desarrollado en cuanto a redes sociales, si bien la interacción es superior que en el resto de organizaciones de atención sanitaria (Busto, 2019).

En el entorno hospitalario la buena reputación se construye a partir de la confianza que los pacientes manifiestan hacia los centros y sus profesionales, al hilo de esto cobra importancia la figura del profesional de la salud, otra de las piezas clave en la imagen que ofrece el hospital. Dichos profesionales se convierten en embajadores de la marca hospitalaria, interactúan con los pacientes e incrementan el interés de estos por el centro y por los tratamientos (George, Rovniav y Kraschnewski, 2013).

Una de las prioridades en la estrategia de comunicación del hospital debería ser formar a estos profesionales de la salud también en destrezas comunicativas en las redes sociales para transmitir correctamente y de manera atractiva la información. Además, sería importante que siguiera la misma línea que el centro para aportar una imagen de marca unificada y con los mismos valores. Los hospitales están apostando por la formación a sus profesionales de la salud en el uso eficaz de las redes sociales (Peluchette, Karl y Coustasse, 2016).

Se ha propuesto que los centros hospitalarios cuenten con una estrategia de comunicación para llevar a cabo su comunicación institucional en redes sociales (Park, Rodgers y Stemmle, 2011). Ello ayudaría a aportar valor a la comunicación ejercida por los hospitales, generaría engagement en sus públicos y formar una imagen consolidada.

Se ha descrito igualmente en torno al refuerzo de dicho engagement que el uso de videos a través de las redes sociales puede tener un mayor impacto en la participación del paciente que las intervenciones basadas en texto, unido al intercambio de información y otras acciones de comunicación (Househ, 
Borycki y Kushniruk, 2014).

El desarrollo del mundo online y el impacto que se observa en los públicos ha generado que sea tan importante para la comunicación institucional de centros hospitalarios la presencia y la buena gestión en redes sociales (Peñafiel, Pastor y Camacho, 2014). Años atrás la prioridad de los hospitales no era mantener una comunicación continua con sus stakeholders en las redes sociales, hoy en día se ha convertido en su razón de ser para identificarse con las necesidades reales de la salud de la población, y se ha señalado su validez como herramienta de comunicación (Griffis et al., 2014).

En este contexto es esencial que el centro hospitalario se convierta en un ente comunicador en todos los sentidos y se adapte a las nuevas exigencias del entorno. El hospital debe adoptar una narrativa transmedia en toda su comunicación, en la que implique directamente a los pacientes haciéndoles formar parte de una comunidad y estrechando los lazos con mediante una comunicación bidireccional.

Es recomendable que los hospitales apuesten sobre todo por soportes multimedia (Gabarron et al., 2013). Algunos utilizan formatos interactivos y audiovisuales para difundir información, ya que de esta manera pueden llegar a un público más amplio y facilitar su asimilación. Una de las tendencias en RRSS más destacadas según el informe "Top Tendencias Digitales" de International Advertising Bureau (IAB Spain, 2019) es precisamente el uso de los contenidos de vídeo y streaming. Youtube sería la red social principal pero también permiten este formato Facebook e Instagram. La red social de Facebook cuenta con 24 millones de usuarios en España frente a 4,9 millones de Twitter (The Social Media Family, 2019)

De las redes sociales más usadas para asuntos de salud, destaca Twitter. Entre sus beneficios estaría la posibilidad de los expertos en salud de publicar diariamente información sanitaria, la posibilidad de relacionarse con otros expertos o investigadores y también el vínculo que se establece con sus públicos y pacientes pudiendo observar el feedback recibido en diferentes temas, lo que les permite saber qué interesa más a sus seguidores (Pershad et al., 2018).

Respecto a Facebook, no solo es usada por personas individuales sino también por organizaciones, para estar en contacto con el mundo. El uso de Facebook puede aportar información sobre salud, dar soluciones y tratamientos, interactuar con pacientes y ayudar a reducir el riesgo de infección. Además, los usuarios pueden compartir problemas y curiosidades con doctores y otros usuarios de Facebook (Adzharuddin y Ramly, 2015).

\subsection{Necesidades y nuevos comportamientos}

El mundo de la salud está compuesto por públicos con capacidad de autogestionarse y de expresar públicamente sus reclamaciones. Un hospital necesita conocer las demandas informativas desu público externo principal: los pacientes. También de su público interno: profesionales del entorno sanitario. Pensar que el efecto de campañas preventivas publicitadas a través de medios de comunicación puede ser una solución, es un error. Todavía no hay evidencia para corroborar ese extremo (Carson et al., 2017). Por otro lado, los segmentos de edad más joven, se han considerado incluso víctimas del sedentarismo y la obesidad por la influencia de los medios (Mazur et al., 2018). La función social de los mass media entraña riesgos, no solo ventajas informativas en la era de internet. En esta misma línea, no es tan claro el efecto beneficioso de los contenidos presentes en los medios de comunicación, sobre todo cuando se centra la atención en un indicador tan claro como es la adherencia al tratamiento (Im y Huh, 2017). No es fácil confiar ciegamente en las noticias médicas, incluso aunque vengan con el mejor de los avales. En ocasiones, las mismas fuentes especializadas son las causantes del "histerismo" clínico. Determinados trabajos clínicos aportan conclusiones que parecen escandalosas y perjudiciales para la salud en primer término y a posteriori resultan ser una interpretación incorrecta, o incluso contradictoria totalmente frente a su aportación inicial (Traish, Vance y Morgentaler, 2017).

Del mismo modo, la relación comunicativa no acaba en la gestión diaria, sino que se prolonga en el tiempo. Más si cabe cuando las personas necesitan atención continuada y una atención diferenciada. Saber mantener los mensajes actualizados e individualizados, con contenidos médicos especializados es una tendencia que se impone progresivamente y sostiene la credibilidad de las organizaciones.

En sociedades donde el proceso de envejecimiento es una realidad, cobra especial importancia los problemas de salud derivados de la neurología. Las personas con enfermedades mentales graves recurren cada vez más a las redes sociales populares, como Facebook, Twitter o YouTube, para compartir sus experiencias sobre enfermedades o buscar el consejo de otras personas con afecciones de salud similares. Esta forma emergente de comunicación no solicitada entre las comunidades en línea que se forman a sí mismas de pacientes y personas con diversos problemas de salud se conoce como apoyo entre pares (peer-to-peer).

En este sentido, se ha observado que precisamente habrá que verificar y determinar si las habilidades aprendidas de sus pares en las redes "on-line" se traducen en mejoras tangibles y significativas en la recuperación, el empleo o el bienestar mental y físico en el mundo "off-line" (Naslund et al., 2016).

Urge por tanto un feedback sostenido: saber qué hacemos mal para poder cambiar. Es ingenuo pensar que nuestros procesos de comunicación no son optimizables. Todo puede mejorar sobre todo de cara a lograr una mejor gestión en los flujos informativos. Desde el prospecto de un producto 
sanitario, hasta la atención en los pasillos de los hospitales, todo necesita adaptarse a los cambios que experimentan las organizaciones. Por eso se precisa testar, auditar y conocer la opinión y el punto de vista de nuestros usuarios. Mucho más que simples compradores de servicios de salud: la razón de ser de la actividad sanitaria. La proactividad de los ingresados en centros sanitarios, por ejemplo, es incluso un factor para tener en cuenta para la mejora de la calidad asistencial, como se ha demostrado (Benham-Hutchinst et al., 2017).

\subsection{Interacción digital y Apps}

La atención sanitaria establece un nuevo vínculo personal entre los interlocutores. Saber explicar en detalle, dar la información actualizada y sin ambigüedades es clave para la satisfacción de cualquier persona usuario de recursos médicos. Los usuarios de los sistemas sanitarios ya vienen con información previa debajo del brazo y con dudas más sofisticadas. La vía de mejora en este caso es la colaboración mutua, especialmente bajo el criterio de los propios especialistas sanitarios (Osei-Frimpong, Wilson y Lemke, 2018). No obstante, es una tarea ardua, ya que entraña considerar de modo individualizado las exigencias de cada paciente y la comprensión de su estado y predisposición a utilizar y aceptar la información recibida (Hibbard, 2017).

Confiar en las aplicaciones médicas (Apps) como una herramienta auxiliar en la mejora de esta colaboración puede ser una opción. Una de las barreras que se presentan para que puedan tener éxito en la consulta médica es que reúnan unos requisitos mínimos de garantía de calidad en sus contenidos, adaptación al paciente y fiabilidad de sus precursores. Un estudio realizado con smartphones en el que participaban padres de familia, asesorados por personal de enfermería, no llegó a ver en las apps una fórmula sencilla y profesional para desarrollar una atención sanitaria profesional (Davis et al., 2017). Igualmente, la evidencia diagnóstica de las aplicaciones de salud disponibles en las tiendas de aplicaciones de Apple y Google es escasa (Buechi et al., 2017). Esta observación resulta ilustrativa para entender que nos encontramos en una fase inicial de la utilidad de los dispositivos móviles.

\section{Objetivo y metodología}

El objetivo principal que se plantea en este trabajo es demostrar que puede plantearse la optimización de la comunicación hospitalaria en redes sociales y lograr estrategias de engagement eficaces. Se propone, por tanto, una metodología de auditoría analítica para optimizar el uso de las redes sociales y el engagement con los públicos, utilizándose como muestra los hospitales de la provincia de Alicante y sus páginas web. Para ello, se analiza la comunicación online de los centros hospitalarios, la repercusión y feedback que tienen los perfiles en las redes sociales Twitter y Facebook. Se compa igualmente la comunicación que realizan los hospitales de gestión pública y los de gestión privada/concertada.

La finalidad de la investigación se enmarca igualmente en la consideración de que la figura del director de comunicación del hospital asume la gestión estratégica de las comunicaciones institucionales para conseguir un posicionamiento de la marca y mejorar las relaciones con todos sus stakeholders, donde dicho director necesita contar con un equipo experto en comunicación institucional con conocimientos de medicina y salud pública (González Pacanowski y Medina Aguerrebere, 2018).

A partir del establecimiento de estos objetivos, se partió de la hipótesis que una correcta gestión de los perfiles corporativos de centros hospitalarios en redes sociales puede contribuir a fortalecer la imagen e identidad digital, tanto de los hospitales públicos como privados. En este sentido, los contenidos multimedia son clave para una efectiva gestión de redes sociales.

Para obtener información estable y legal sobre los hospitales públicos de la provincia de Alicante, se ha definido inicialmente una muestra atendiendo al listado oficial de Centros Hospitalarios Públicos Valencianos por provincias de la Conselleria de Sanitat de la Generalitat Valenciana. Para extraer datos sobre las redes sociales y web propia de cada centro, tanto de gestión pública como privada, se ha recurrido a la base de datos ObservaTICs del grupo de Tecnologías para la salud y Bienestar del Instituto ITACA (ITACA-TSB) de la Universidad Politécnica de Valencia.

La descripción del engagement o grado de interacción se estableció mediante un análisis cuantitativo, identificando los seguidores, contenido aportado y el feedback recibido en las redes sociales Twitter y Facebook. Se ha realizado la obtención de datos mediante las herramientas de analítica FollowerWonk, Twitonomy y ObservaTICs (ITACA-TSB) para Twitter y la herramienta Likealyzer para el análisis de Facebook. Para identificar contenido con más engagement se ha empleado la herramienta Socialbakers.

FollowerWonk1, Twitonomy², las métricas de ObservaTICs (ITACA-TSB) para Twitter, y Likealyzer ${ }^{3}$ empleada para el análisis de Facebook, son herramientas de uso gratuito y online. Socialbakers ${ }^{4}$ es gratuita de uso temporal. Para el análisis de las páginas web de los centros hospitalarios se ha utilizado la herramienta SimilarWeb ${ }^{5}$, que permite realizar comparaciones entre distintas webs para ver el impacto que tienen cada una.

Mediante Followerwonk se han descrito los indicadores de followers únicos, años de indexación, retweets y autoridad social (Social Authority). A través de Lykealyzer es posible conocer tres indicadores: número de personas que dialogan: PTAT (People Talking About This), número de likes y el ratio de engagement calculado dividiendo el PTAT entre el número total de likes. Socialbakers se ha utilizado para obtener puntualmente ejemplos del engagement de post en Facebook 


\begin{tabular}{|c|c|c|c|c|}
\hline Centro Sanitario & Localización & Facebook & Twitter & Web \\
\hline Clínica Vistahermosa S.A* & Alicante & & & • \\
\hline Hospital Clínica Benidorm* & Benidorm & • & • & • \\
\hline Hospital de Dénia & Dénia & • & • & - \\
\hline Hospital de la Marina Baixa & Vila Joiosa & - & - & - \\
\hline Hospital General de Elche & Elx & • & • & • \\
\hline Hospital General de Elda & Elda & - & • & - \\
\hline Hospital General de Alicante & Alicante & $\cdot$ & $\cdot$ & $\cdot$ \\
\hline Hospital IMED Levante* & Elx & - & & - \\
\hline Hospital Internacional Medimar* & Alicante & $\cdot$ & $\cdot$ & $\cdot$ \\
\hline Hospital La Pedrera & Dénia & $\cdot$ & $\cdot$ & $\cdot$ \\
\hline Hospital IMED Elche* & Elx & - & & - \\
\hline Hospital Universitario Torrevieja & Torrevieja & - & $\cdot$ & - \\
\hline Hospital Quiron Torrevieja* & Torrevieja & & & - \\
\hline Hospital Universitario de Vinalopó & Elx & • & • & • \\
\hline Hospital Universitario de Sant Joan & Alicante & • & • & - \\
\hline Hospital Vega Baja & Orihuela & • & • & - \\
\hline Hospital Virgen de los Lirios & Alcoi & & - & - \\
\hline
\end{tabular}

Tabla 1. Muestra de hospitales y recursos online. Autoría a partir de ObservaTICs. (*hospitales privados) y elaboración propia.

midiendo interacciones, reacciones, comentarios y compartir. No se han explorado las opciones de pago profesionales de las herramientas.

Se descartó Youtube dada su baja frecuencia dialógica en el ámbito propuesto, a pesar de su carácter de repositorio multimedia evidente.

Tras analizar las redes sociales de 17 centros hospitalarios (Twitter y Facebook), así como las páginas web propias, se han interpretado los resultados obtenidos, gracias a las herramientas citadas anteriormente durante tres meses continuados (10-XII-2019/31-XII-2019). El análisis web se realizó entre los meses de febrero de 2019 hasta mayo de 2019. El hecho de elegir la provincia de Alicante obedece a que proporciona una muestra que recoge variabilidad de perfiles institucionales, al mismo tiempo que cuenta con un porcentaje significativo de centros acreditados por su calidad asistencial y organizativa a cargo de la Joint Comission norteamericana (Joint Comission) ${ }^{6}$. Se contabilizaron las redes sociales de las que dispone cada centro hospitalario. Todas disponen de página web propia y la gran mayoría tiene perfil en Facebook o Twitter (Tabla 1).

\section{Resultados}

Tras un análisis cuantitativo de los seguidores en los perfiles de Twitter, se observa (tabla 2) que los tres centros con más seguidores y que más feedback reciben son el Hospital Universitario del Vinalopó de Elche (3.633), el Hospital Universitario de Torrevieja (3.039) y el Hospital de Dénia (2.579). Estos tres centros ofrecen cobertura sanitaria pública y están gestionados por la compañía Ribera Salud.

\begin{tabular}{|l|l|l|}
\hline \multicolumn{1}{|c|}{ Centro Sanitario } & Facebook & Twitter \\
\hline Clínica Vistahermosa S.A & & \\
\hline Hospital Clínica Benidorm & 2.565 & 247 \\
\hline Hospital de Dénia & 4.976 & 2.579 \\
\hline Hospital de la Marina Baixa & 1.911 & 1.484 \\
\hline Hospital General de Elche & 2.535 & 1.465 \\
\hline Hospital General de Elda & 2.190 & 475 \\
\hline Hospital General de Alicante & 2.710 & 2.047 \\
\hline Hospital IMED Levante & 4.936 & \\
\hline Hospital Internacional Medimar & 11.998 & 883 \\
\hline Hospital La Pedrera & 1.667 & 823 \\
\hline Hospital IMED Elche & 4.922 & \\
\hline Hospital Universitario Torrevieja & 5.627 & 3.039 \\
\hline Hospital Quirón Torrevieja & & \\
\hline Hospital Universitario de Vinalopó & 7.006 & 3.633 \\
\hline Hospital Universitario de Sant loan & 2.777 & 1.871 \\
\hline Hospital Vega Baja & 2.893 & 1.381 \\
\hline Hospital Virgen de los Lirios & & 258 \\
\hline
\end{tabular}

Tabla 2. Seguidores en Facebook y Twitter en 2019. Elaboración propia

Las redes sociales de estos centros mantienen una actividad diaria en Twitter, con una media de una y dos publicaciones al 
día y porcentajes de retweets a otros usuarios entre el $26 \%$ y el $28 \%$.

Perfiles de gestión pública como el del Hospital General de Alicante cuentan con un gran número de seguidores (2.047), si bien no alcanza la media de un tuit por día. No obstante, interactúa con los usuarios llegando al 55\% de retweets realizados. El Hospital Universitario de San Juan roza los dos mil seguidores también (1.871) y refleja una actividad significativa en el perfil con una media de 1,22 tuits por día y un 68\% de retweets a otras cuentas, generando un feedback continuo con los usuarios.

Se ha observado que los dos hospitales de la ciudad de Alicante en los meses analizados obtuvieron un incremento de los seguidores, con tendencia mantenida al alza. Destacan también entre los hospitales gestionados por la Generalitat por su continua actividad en Twitter; el Hospital General de Elche, que contaba con una media de 2,92 publicaciones al día, siendo el hospital con más actividad y el Hospital Vega Baja de Orihuela, que realizaba una media de 1,39 publicaciones al día. Estos dos hospitales, además, superaban en el momento del análisis la franja de los mil seguidores. El Hospital General de Elda contaba con un promedio de 2,70 publicaciones por día, a pesar de ello, es el tercer centro hospitalario con menos seguidores en Twitter.

De los seis centros privados de la provincia, tan sólo dos de ellos cuentan con un perfil propio en Twitter, como son el Hospital Internacional Medimar y Hospital Clínica Benidorm. Tras analizar sus seguidores y su actividad en la red social, se observa que el hospital de Benidorm es el centro con menos seguidores en Twitter (247) y destaca su baja actividad, registrando en este sentido semanas sin publicar.

En el caso del Hospital Internacional Medimar, gestionado por Vithas Alicante, el perfil cuenta con un mayor número de seguidores que el anterior (883). Comparativamente supone una cantidad reducida teniendo en cuenta su localización en la capital de la provincia. Su actividad en Twitter es escasa y no realiza interacciones con sus seguidores. No obstante, fue uno de los primeros centros de la provincia en tener perfil de Twitter, hace casi nueve años.

Los datos de actividad de los perfiles de Twitter (tabla 3) se han obtenido mediante la herramienta online Twitonomy que genera medias y porcentajes de las publicaciones e interacciones realizadas desde la fecha de creación de los perfiles hasta la actualidad. El promedio de la actividad diaria, extraído de las herramientas analíticas, muestra que los hospitales de la provincia con perfil propio en Twitter, registran medias con valores de hasta 2,92 de media de publicaciones como es el caso del Hospital General de Elche.

\begin{tabular}{|l|c|}
\hline \multicolumn{1}{|c|}{ Centro Sanitario } & $\begin{array}{c}\text { Medias publicaciones } \\
\text { Twitter }\end{array}$ \\
\hline Clínica Vistahermosa S.A & \\
\hline Hospital Clínica Benidorm & 0,9 \\
\hline Hospital de Dénia & 1,07 \\
\hline Hospital de la Marina Baixa & 0,98 \\
\hline Hospital General de Elche & 2,92 \\
\hline Hospital General de Elda & 2,7 \\
\hline Hospital General de Alicante & 0,68 \\
\hline Hospital IMED Levante & \\
\hline Hospital Internacional Medimar & 0,74 \\
\hline Hospital La Pedrera & 0,8 \\
\hline Hospital IMED Elche & \\
\hline Hospital Universitario Torrevieja & 1,72 \\
\hline Hospital Quiron Torrevieja & \\
\hline Hospital Universitario de Vinalopó & 2,02 \\
\hline Hospital Universitario de Sant Joan & 1,22 \\
\hline Hospital Vega Baja & 1,39 \\
\hline Hospital Virgen de los Lirios & 1,09 \\
\hline Total: & 1,40 \\
\hline
\end{tabular}

Tabla 3. Promedios de tuits por día en 2019. Elaboración propia.

Tras el análisis cuantitativo de seguidores y la actividad de los perfiles propios de Facebook, existe cierto contraste con los datos obtenidos del análisis de Twitter.

Una de las grandes diferencias se encuentra en el perfil del Hospital Internacional Medimar (tabla 2), gestionado por Vithas Alicante, el cual cuenta con casi doce mil seguidores en su cuenta de Facebook (11.998), sin embargo en Twitter recibe escaso feedback de los seguidores.

Los principales perfiles con más seguidores son el del Hospital Universitario del Vinalopó (7.006), el Hospital Universitario de Torrevieja (5.625) y el Hospital de Dénia (4.976), seguidos por los dos hospitales gestionados por IMED, en Elche; IMED Levante (4.936) e IMED Elche (4.922).

La red social Facebook en los hospitales privados o de gestión concertada representa la que más seguidores y likes tienen en sus perfiles (tabla 2). El resto de perfiles, gestionados por centros públicos de la Generalitat, no superan los tres mil seguidores y las cifras entre ellos son bastante similares, ninguno destaca sobre el resto.

En lo relativo a la actividad de cada centro en Facebook (tabla 4), el centro con mayor actividad es el Hospital Universitario de Torrevieja con una media de 1,6 publicaciones al día, seguido por el Hospital Internacional Medimar con 1,4 post diarios. Contrasta con su actividad diaria en Twitter, donde no alcanza 
al tuit por día.

\begin{tabular}{|l|c|}
\hline \multicolumn{1}{|c|}{ Centro Sanitario } & $\begin{array}{c}\text { Media Publicación } \\
\text { Facebook }\end{array}$ \\
\hline Hospital Clínica Benidorm & 0,4 \\
\hline Hospital de Dénia & 0,4 \\
\hline Hospital de la Marina Baixa & 0,1 \\
\hline Hospital General de Elche & 1,1 \\
\hline Hospital General de Elda & 0 \\
\hline Hospital General de Alicante & 0,3 \\
\hline Hospital IMED Levante & 0,2 \\
\hline Hospital Internacional Medimar & 1,4 \\
\hline Hospital La Pedrera & 0,3 \\
\hline Hospital IMED Elche & 0,3 \\
\hline Hospital Universitario Torrevieja & 1,6 \\
\hline Hospital Universitario de Vinalopó & 1,1 \\
\hline Hospital Universitario de Sant loan & 0,5 \\
\hline Hospital Vega Baja & 1,1 \\
\hline Total: & 0,6 \\
\hline
\end{tabular}

Tabla 4. Promedios de publicaciones en Facebook por día en 2009. Elaboración propia

El Hospital Universitario del Vinalopó (gráfico 2), el segundo en número de seguidores, publica una media de 1,1 post al día en Facebook. Los hospitales públicos de Elche y Orihuela tienen también una media de publicaciones diarias de 1,1, pero con un volumen de seguidores inferior.

El resto de hospitales tiene una escasa actividad en Facebook, no llegando a la media de un post diario. En concreto, llama la atención la escasa actividad de los centros gestionados por IMED, si bien cuenta con bastantes seguidores.

La diferencia que encontramos entre los análisis de Facebook y Twitter es la escasa actividad en Facebook de los centros hospitalarios analizados. El promedio total de publicaciones que realizan los perfiles analizados no llega al post diario $(0,65)$.

En conclusión, en Facebook los hospitales analizados de la provincia de Alicante registran menos actividad que en sus perfiles de Twitter, pese a que el número de usuarios es mayor en todos los casos.

Atendiendo a los perfiles de Facebook con mayor peso, podemos observar que el Hospital Universitario de Torrevieja (gráfico 2), además de tener una actividad destacable, realiza publicaciones de calidad. El 57\% de contenido incluye imágenes. Contabiliza la publicación de 25 vídeos nativos de Facebook.
El Hospital Internacional Medimar cuenta con una actividad diaria de calidad, con un $80 \%$ de imágenes en sus posts y una interacción con 4 vídeos nativos en Facebook. El tercero es el Hospital Universitario del Vinalopó que cuenta con un 56\% en contenido de imágenes y con 15 vídeos nativos de Facebook.

El contenido audiovisual es el que más impacto genera en los seguidores, por lo que va a ser determinante a la hora de gestionar un perfil en cualquier red social. Los tres centros hospitalarios con más seguidores en Facebook son los que más utilizan en sus publicaciones contenido audiovisual.

Esta observación lleva a plantear que en breve plazo presumiblemente aumente la producción de contenidos con este formato y contenido. No sólo por su atractivo y diferencial de marca que proporciona, si no sobre todo por las posibilidades de educación sanitaria y colaboración entre los públicos como son los propios pacientes o profesionales sanitarios.

Los resultados obtenidos del análisis cuantitativo ponen de manifiesto varias consideraciones.

El primer matiz lo encontramos en los perfiles de la red social Twitter. Según se comprueba, de los seis centros hospitalarios privados, tan sólo dos tienen perfil en Twitter (Hospital Clínica Benidorm y Hospital Internacional Medimar), con resultados poco relevantes comparativamente tanto en número de seguidores como en la actividad registrada (Tablas 2 y 3 ).

Entre los tres hospitales con mejores cifras de seguidores y feedback recibido, se encuentran los pertenecientes al Grupo Ribera Salud, hospitales de gestión concertada: Hospital Universitario del Vinalopó, Hospital Universitario Torrevieja y el Hospital de Denia (Tabla 2). Son hospitales que ofrecen cobertura pública, pero la gestión es llevada a cabo por empresas privadas, por lo tanto la comunicación es gestionada por la empresa, contando con mayores medios.

Por otro lado, cabría destacar que la mayoría de los hospitales de la Generalitat en sus perfiles de Twitter, a pesar de contar con menos seguidores, arrojan cifras de actividad superando en algunos casos (Hospital General de Elche y Elda) a los concertados (Tabla 3), si bien la demanda por cupo poblacional sanitario asignado por la Consejería de Sanidad de la Generalitat de Valencia considerado como sesgo podría explicar este hecho. En contraposición, tenemos a Facebook que confirma en su totalidad las hipótesis planteadas. Destaca en número de seguidores el Hospital Internacional Medimar de gestión privada.

La mayoría de hospitales privados tienen resultados relevantes en número de seguidores y likes, también los tres de gestión concertada (Tabla 2).

Se aprecian, por tanto, diferencias notables con las cifras de los hospitales públicos. Los hospitales privados y concertados superan también en promedios de actividad en Facebook 
a los públicos (Tabla 4). Continuando con el análisis cuantitativo del contenido de los perfiles de Facebook (Likealyzer), se corrobora la gran importancia de los contenidos audiovisuales e interactivos para generar feedback con los seguidores y fomentar una buena reputación. Es por ello que los perfiles que más imágenes y vídeos nativos de Facebook comparten, tienen mejores resultados en números de seguidores e interacción (gráfico 1). El nivel de actividad y contenido en Facebook del Hospital Universitario de Torrevieja, Hospital del Vinalopó, ambos pertenecientes al grupo Ribera Salud, y Hospital Internacional Medimar sirven para correlacionar el uso de vídeo nativo con el ratio de engagement, más elevado cuanto mayor es la producción audiovisual propia. Igualmente, el PTAT y el volumen de likes se incrementa de modo evidente.

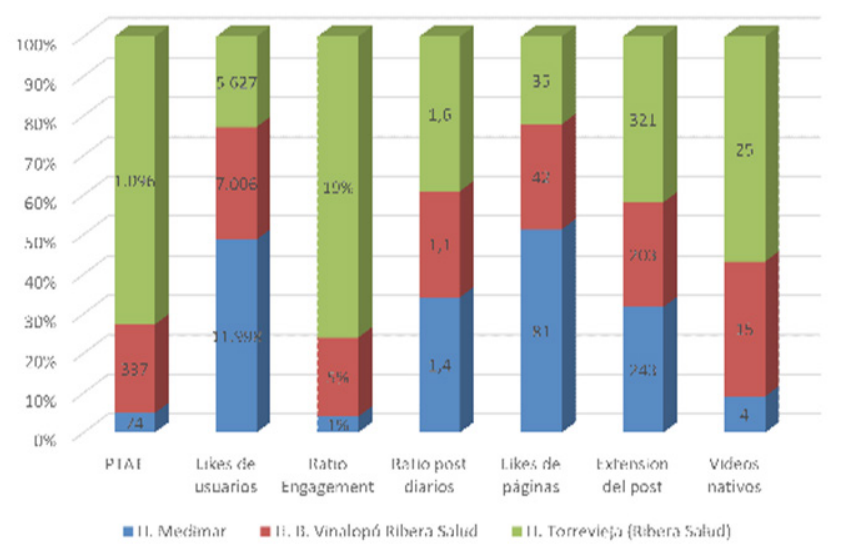

Gráfico 1. Comparativo de indicadores de Likelyaser. Elaboración propia.

El formato que mejor engagement produce a través de Facebook combina texto imagen, infografía y vídeo, como se aprecia en las métricas específicas del Grupo Ribera Salud. Se utiliza información práctica y con valor testimonial (figuras 1 , 2 y 3$)$.

Respecto a la gestión de redes sociales corporativas en Twitter, los hospitales privados y concertados superan a los públicos notablemente en seguidores y calidad de contenido en la gran mayoría de casos comparados (gráficos 1 y 2)ㄱ. En Followerwonk se han escogido dos perfiles de Twitter, para cada ejemplo de centros hospitalarios que pertenecen a la misma localidad; uno de ellos es de gestión pública y el otro de gestión concertada.

Por otra parte, tras analizar los tuits de las tres cuentas con más actividad se observa que los temas que más repercusión y feedback consiguen son las noticias que publican con información sobre salud general y las publicaciones de días internacionales relacionados con la salud.

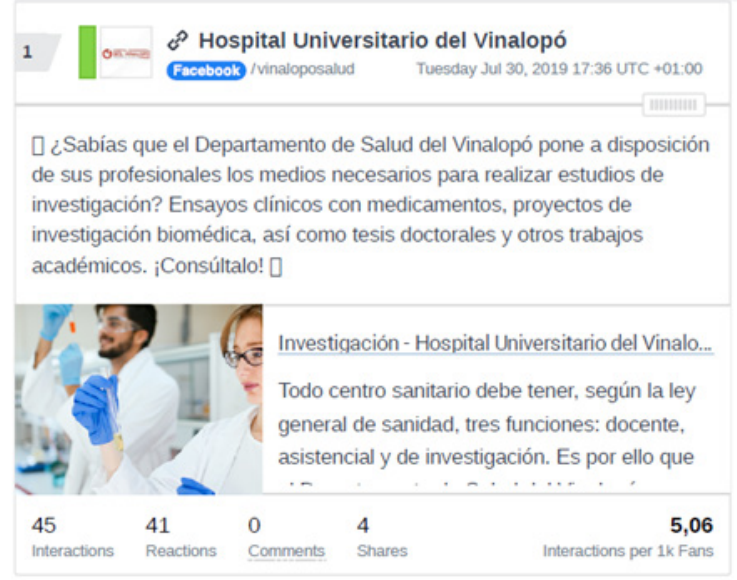

Figura 1. Post institucional en Facebook grupo Ribera.

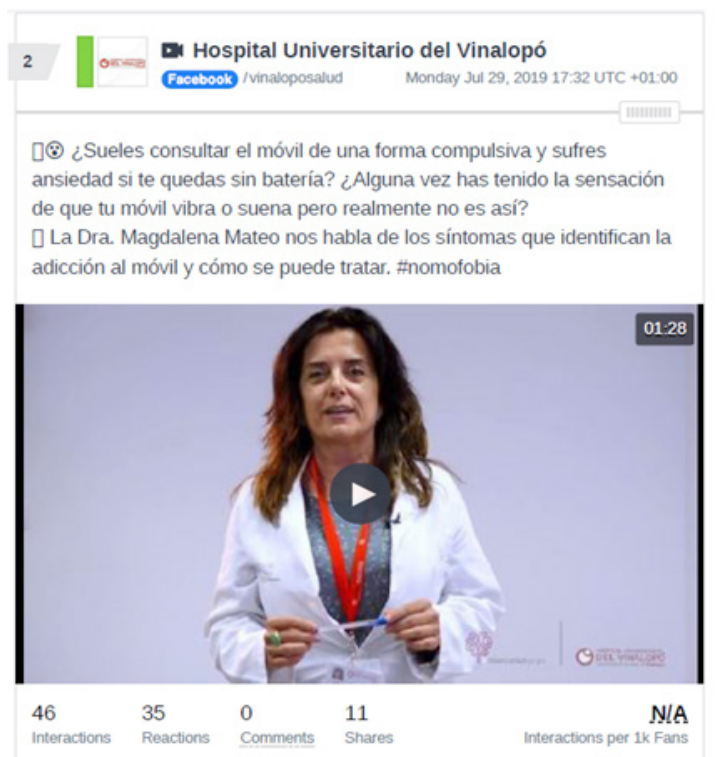

Figura 2. Post de interés sanitario en Facebook del Grupo Ribera.

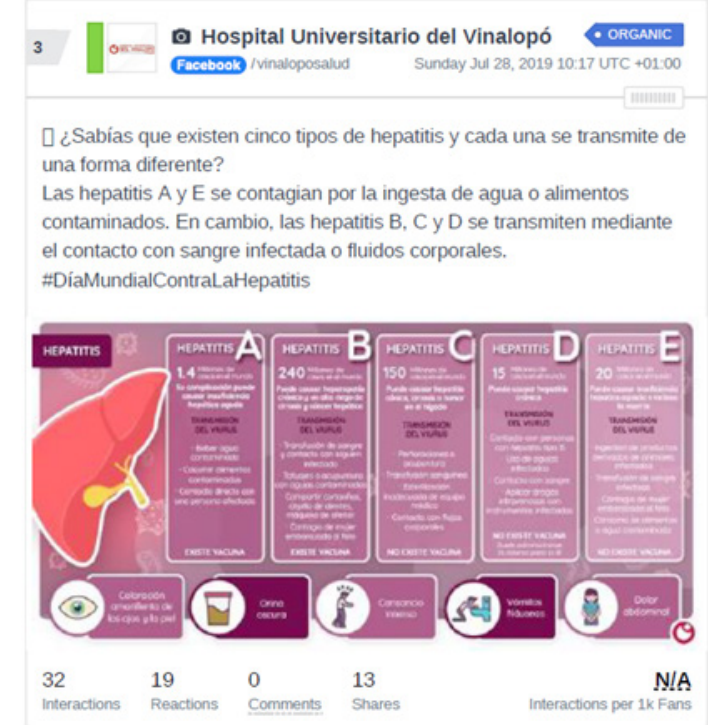

Figura 3. Post formativo en Facebook del Grupo Ribera. 


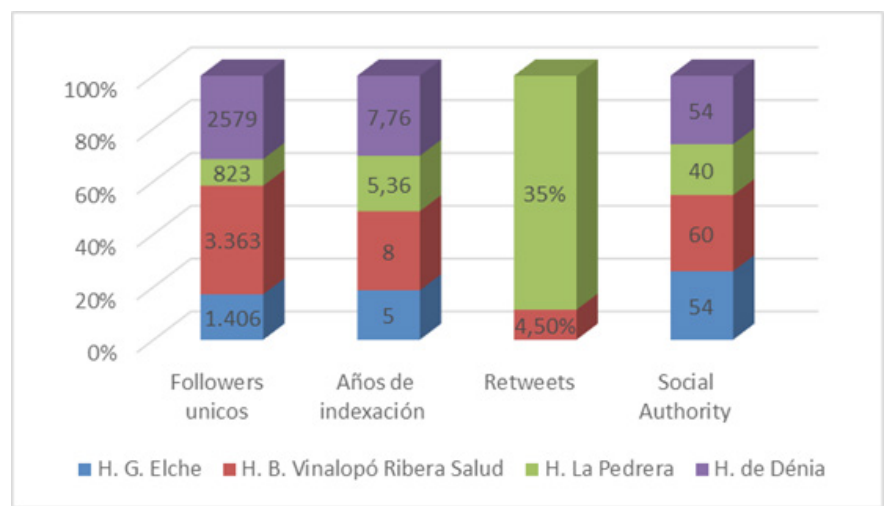

Gráfico 2. Comparativo followers en Twitter. Elaboración propia a partir de Followerwonk.

\subsection{Tráfico web}

Para tener una idea del posicionamiento web de las corporaciones sanitarias analizadas, se ha elaborado un ránking que permite dar una idea del alcance en internet. En cuanto al análisis de las páginas web de los centros hospitalarios, se recaban datos de visitas a las páginas web propias de los diecisiete centros Hospitalarios analizados. En este caso, las visitas contabilizadas abarcan desde el mes de febrero de 2019 hasta mayo de 2019. Se ha utilizado la herramienta de análisis web SimilarWeb, que contabiliza las visitas mensuales y realiza rankings globales y estatales de las páginas más influyentes.

La página web con más visitas es la del Hospital Quirón Torrevieja (tabla 5), con 3,942 millones de visitas. Ocupaba en el momento del análisis el puesto 972 en el ranking de España de páginas web.

El Hospital Internacional Medimar de Vithas Alicante registró 533.611 visitas en los tres últimos meses analizados en el 2019. En el caso del Hospital Clínica Vistahermosa, gestionado por HLA, se contabilizaron 334.733 visitas a su página web en el mismo periodo de tiempo. Estos tres centros de gestión privada cuentan con una página web que pertenece a la empresa que los gestiona. Su audiencia es nacional e internacional, por lo que son páginas con influencia en toda España y también en Latinoamérica.

\begin{tabular}{|l|l|}
\hline \multicolumn{1}{|c|}{ Centro sanitario } & \multicolumn{1}{|c|}{$\begin{array}{c}\text { Visitas (no visitante } \\
\text { único) a la Web }\end{array}$} \\
\hline Clínica Vistahermosa S.A & 334.733 \\
\hline Hospital Clínica Benidorm & 172.720 \\
\hline Hospital de Dénia & 79.507 \\
\hline Hospital de la Marina Baixa & 19,092 \\
\hline Hospital General de Elche & 30.001 \\
\hline Hospital General de Elda & 20.486 \\
\hline Hospital General de Alicante & 37.910 \\
\hline Hospital IMED Levante & 72.926 \\
\hline
\end{tabular}

\begin{tabular}{|l|l|}
\hline Hospital Internacional Medimar & 533.611 \\
\hline Hospital La Pedrera & $<5.000$ \\
\hline Hospital IMED Elche & 39.177 \\
\hline Hospital Universitario Torrevieja & 269.347 \\
\hline Hospital Quirón Torrevieja & $3.942 \mathrm{M}$ \\
\hline Hospital Universitario de Vinalopó & 180.893 \\
\hline Hospital Universitario de Sant Joan & 25.995 \\
\hline Hospital Vega Baja & 43.137 \\
\hline Hospital Virgen de los Lirios & $<5.000$ \\
\hline
\end{tabular}

Tabla 5. Visitas totales a la página web en 2019. Elaboración propia

El Hospital Clínica Benidorm contabilizó 172.720 visitas. Los dos hospitales gestionados por Vinalopó Salud, Hospital Universitario Torrevieja y Hospital Universitario de Vinalopó contabilizaron 269.347 y 180.893 visitas respectivamente en el trimestre referido anteriormente.

Estos tres últimos disponen de página web del propio centro, independiente de la web de la empresa que los gestiona. Los seis centros con más visitas a su página web son de gestión privada o concertada.

El resto de hospitales de gestión pública registraron menos visitas y no contabilizan en el ranking de SimilarWeb, ya que sus páginas web son departamentos dentro de la página web de la Conselleria de Sanitat de la Generalitat Valenciana.

\section{Conclusiones}

Podemos decir en primer lugar y respecto al objetivo principal de este trabajo, que es posible encontrar fórmulas para mejorar el tráfico y el posicionamiento de los hospitales en una comunicación a través de redes sociales, basándonos en la experiencia de la provincia de Alicante.

El éxito de la optimización ámbito comunicativo de las redes sociales para logar mayor eficiencia implica sin embargo nuevas estrategias y objetivos. Destaca, por tanto, la importancia de los contenidos multimedia una vez más y de contenidos que generen un interés en el usuario y que le aporten valor. De esta manera podrán conseguirse mejores resultados en los perfiles corporativos de los centros hospitalarios que repercutan positivamente en la imagen y reputación de los mismos, atendiendo al ratio de engagement.

A día de hoy el dispositivo más utilizado por los usuarios en redes sociales y por la mayor parte de la población es el móvil, de ahí la importancia del Mobile Marketing y que en comunicación institucional, específicamente en el ámbito sanitario, no está explotado, salvo casos puntuales. Es por tanto importante que los contenidos que ofrezca el hospital en su página web también sean de diseño responsive, es decir adaptable a 
todos los dispositivos.

Las redes sociales permiten tener una plataforma que facilita la comunicación y la interacción, donde se puede compartir información sobre cuidados de la salud como consejos, prevención de enfermedades, tratamientos posibles y prescripciones y citas con especialistas. Para lograr este objetivo, la planificación de un plan de contenidos segmentada por públicos tanto externos como internos de los centros hospitalarios es clave

En esta investigación las herramientas analíticas utilizadas para observar y comparar los perfiles corporativos, de Twitter y Facebook, de los hospitales analizados, así como las páginas web de estos, se reafirman como una fórmula válida para medir el dialogo social y los procesos comunicativos que se dan en las redes sociales por parte de los centros hospitalarios de la provincia de Alicante.

Se demuestra, en este sentido, que hay diferencias notables en la gestión de las redes sociales y la comunicación entre los centros gestionados por la Generalitat de la Comunidad Valenciana y los centros gestionados por empresas privadas; teniendo estos últimos mayores medios y más eficaces, lo que podría considerarse un valor para proyectar mejor imagen y reputación. En este sentido, existe evidencia por tanto que una mayor actividad en redes sociales se correlaciona con excelencia en la calidad asistencial, como sucede en el caso de los centros concertados del Grupo Ribera Salud. No obstante, la diferenciación entre naturaleza pública y privada no debería ser un condicionante para el desarrollo de contenidos de calidad que refuercen los vínculos paciente-médico y paciente-hospital.

Esta investigación pretende, en síntesis, ser una muestra representativa de la relación que tienen los Hospitales de Alicante con las redes sociales y de qué manera podría mejorarse para establecer una comunicación con sus stakeholders más eficaz y consolidar su imagen de marca corporativa. Para lograr ese objetivo, el seguimiento y atención efectiva de los pacientes mediante una interacción correcta en las redes sociales es hoy imprescindible.

\section{Notas al final}

1. Web de FollowerWonk: https://followerwonk.com/

2. Web de Twitonomy: https://www.twitonomy.com/

3. Web de Lykealyzer: https://likealyzer.com/

4. Web de Socialbakers: https://www.socialbakers.com/

5. Web de Similarweb: https://www.similarweb.com/

6. Centros españoles acreditados por la Joint Comission. Ver en http://www.acreditacionfada.org/index.php?page=organizaciones-acreditadas

7. En Followerwonk se han escogido dos perfiles de Twitter, para cada ejemplo de centros hospitalarios que pertenecen a la misma localidad; uno de ellos es de gestión pública y el otro de gestión concertada.

\section{Financiación}

Esta investigación forma parte del Plan Estatal de Investigación Científica y Técnica y de Innovación 2017-2020. Proyectos de I+D+i «Retos investigación» (RTI2018-095714-B-C21). Ministerio de Educación, Gobierno de España.

\section{Referencias}

Adzharuddin, N. A. y Ramly, N. M. (2015). Nourishing Healthcare Information over Facebook. Procedia. Social and Behavioral Sciences, 172 , 383-389. https://doi.org/10.1016/j.sbspro.2015.01.384

Becerra Muñoz, E., Reina Estévez, J., Mas, V. y Salvador, J. (2015). Comunicación e imagen de los servicios sanitarios. Revista Prisma Social, (14), 1-28.

Benham-Hutchins, M., Staggers, N., Mackert, M., y Johnson, A. H. (2017). "I want to know everything": a qualitative study of perspectives from patients with chronic diseases on sharing health information during hospitalization. BMC health services research, 17(1), 529.

Buechi, R., Faes, L., Bachmann, L., Thiel, M., Bodmer, N., Schmid, M. y Lienhard, K. (2017). Evidence assessing the diagnostic performance of medical smartphone apps: a systematic review and exploratory meta-analysis. BMJ open, 7(12), e018280. https://doi.org/10.1136/ bmjopen-2017-018280

Busto-Salinas, L. (2019). Sanidad y redes sociales: ¿Que entidades son mas activas y con cuales interactua mas el publico? El Profesional de la Informacion, 28(2). https://doi.org/10.3145/epi.2019.mar.15

Carson, K. V., Ameer, F., Sayehmiri, K., Hnin, K., Van Agteren, J., Sayehmiri, F. y Smith, B. (2017). Mass media interventions for preventing smoking in young people. The Cochrane Library, 2(6), CD001006. https://doi.org/10.1002/14651858.CD001006.pub3

Costa, C., Túñez, M. y Videla. J. (2016). Hospitales españoles en la web social. Gestión de Facebook y Twitter por el Hospital Sant Joan de Déu (Barcelona). Revista latina de comunicación social, (71), 1108-1130. https://doi.org/10.4185/RLCS-2016-1137

Davis, D. W., Logsdon, M. C., Vogt, K., Rushton, J., Myers, J., Lauf, A. y Hogan, F. (2017). Parent Education is Changing: A Review of Smartphone Apps. MCN: The American Journal of Maternal/Child Nursing, 42(5), 248-256. https://doi.org/10.1097/NMC.0000000000000353

Gabarron, E., Fernandez-Luque, L., Armayones, M. y Lau, A. Y. (2013). Identifying Measures Used for Assessing Quality of YouTube Videos with Patient Health Information: A Review of Current Literature. Interactive Journal of Medical Research, 2(1), e6. https://doi.org/10.2196/ ijmr.2465

George, D. R., Rovniak, L. S. y Kraschnewski, J. L. (2013). Dangers and Opportunities for Social Media in Medicine. Clinical Obstetrics and Gynecology, 56(3), 453-462. https://doi.org/10.1097/ grf.0b013e318297dc38

Glover, M., Khalilzadeh, O., Choy, G., Prabhakar, A. M., Pandharipande, P. V. y Gazelle, G. S. (2015). Hospital evaluations by social media: a comparative analysis of Facebook ratings among performance outliers. Journal of General Internal Medicine, 30(10), 1440-1446. https://doi.org/10.1007/s11606-015-3236-3

González Pacanowski, A. y Medina Aguerrebere, P. (2018). El rol de las redes sociales en la difusión de la identidad corporativa de los hospitales españoles. Revista de la Asociación Española de Investigación 
de la Comunicación, 5(10), 30-38. https://doi.org/10.24137/raeic.5.10.5

Griffis, H. M., Kilaru, A. S., Werner, R. M., Asch, D. A., Hershey, J. C., Hill, S. y Merchant, R. M. (2014). Use of social media across US hospitals: descriptive analysis of adoption and utilization. Journal of Medical Internet Research, 16(11), e264. https://doi.org/10.2196/jmir.3758

Hibbard, J. H. (2017). Patient activation and the use of information to support informed health decisions. Patient Education and Counseling, 100(1), 5-7. https://doi.org/10.1016/j.pec.2016.07.006

Househ, M., Borycki, E., y Kushniruk, A. (2014). Empowering patients through social media: the benefits and challenges. Health informatics journal, 20(1), 50-58. https://doi.org/10.1177/1460458213476969

IAB Spain (2019). Top Tendencias Digitales. Recuperado de https:// iabspain.es/wp-content/uploads/top_tendencias_digitales_iab_ spain_2019.pdf

Im, H. y Huh, J. (2017). Does health information in mass media help or hurt patients? Investigation of potential negative influence of mass media health information on patients' beliefs and medication regimen adherence. Journal of Health Communication, 22(3), 214-222. https://doi.org/10.1080/10810730.2016.1261970

Kumar, P. N., Jacob, A. y Thota, S. (2014). Impact of Healthcare Marketing and Branding on Hospital Services. International Journal of Research Foundation of Hospital and Health Care Administration, (2), 19-24. https://doi.org/10.5005/jp-journals-10035-1010

López-Bolás, A., Valderrama-Santomé, M., y Di-Virgilio, F. (2019). Claves del éxito para la viralización de contenidos de salud. El caso de las redes sociales del Hospital Povisa. Profesional de la Información, 28(5). https://doi.org/10.3145/epi.2019.sep.02

Martinez-Millana, A., Fernandez-Llatas, C., Bilbao, I. B. Salcedo, M. T. y Salcedo, V. T. (2017). Evaluating the social media performance of hospitals in Spain: A longitudinal and comparative study. Journal of Medical Internet Research, 19(5), e181. https://doi.org/10.2196/ jmir.6763

Mazur, A., Caroli, M., Radziewicz-Winnicki, I., Nowicka, P., Weghuber, D., Neubauer, D. y Hadjipanayis, A. (2018). Reviewing and addressing the link between mass media and the increase in obesity among European children: The European Academy of Paediatrics (EAP) and The European Childhood Obesity Group (ECOG) consensus statement. Acta Paediatrica, 107(4), 568-576. https://doi.org/10.1111/apa.14136

Naslund, J. A., Aschbrenner, K. A., Marsch, L. A. y Bartels, S. J. (2016). The future of mental health care: peer-to-peer support and social media. Epidemiology and Psychiatric Sciences, 25(2), 113-122. https:// doi.org/10.1017/S2045796015001067

Naveen, K., Anil, J., y Smruthi, T. (2014). Impact of healthcare marketing and branding on hospital services. International Journal of Research Foundation of Hospital \& Healthcare Administration, 2(1), 19-24. https://doi.org/10.5005/jp-journals-10035-1010

Observatorio Nacional de las Telecomunicaciones y de la Sociedad de la Información (2016). Los ciudadanos ante la e-Sanidad. Opiniones y expectativas de los ciudadanos sobre el uso y la aplicación de las TIC en el ámbito sanitario. Recuperado de https://www.ontsi.red.es/ ontsi/sites/ontsi/files/los_ciudadanos_ante_la_e-sanidad.pdf

Osei-Frimpong, K., Wilson, A. y Lemke, F. (2018). Patient co-creation activities in healthcare service delivery at the micro level: The influence of online access to healthcare information. Technological Forecasting and Social Change, (126), 14-27. https://doi.org/10.1016/j. techfore.2016.04.009

Park, H., Rodgers, S. y Stemmle, J. (2011). Health Organizations' Use of
Facebook for Health Advertising and Promotion. Journal of Interactive Advertising, 12(1), 62-77. https://doi.org/10.1080/15252019.2011.1072 2191

Peluchette, J. V., Karl, K. A. y Coustasse, A. (2016). Physicians, patients, and Facebook: Could you? Would you? Should you? Health Marketing Quarterly, 33(2), 112-126. https://doi.org/10.1080/07359683.2016.116 6811

Peñafiel Saiz, C., Pastor González, J. M. y Camacho Markina, I. (2014). La información de salud en el universo web dirigida a la juventud: entre profiláctica y educativa. En Carmen Peñafiel y Lázaro Echegaray (Coords.), Estudios de Comunicación y Salud, CAC 63. Sociedad Latina de Comunicación Social.

Pershad, Y., Hangge, P., Albadawi, H. y Oklu, R. (2018). Social Medicine: Twitter in Healthcare. Journal of Clinical Medicine, 7(6), 121. https:// doi.org/10.3390/jcm7060121

Rando Cueto, D., Paniagua Rojano, F. y De las Heras Pedrosa, C. (2016). Influence factors on the success of hospital communication via social networks. Revista Latina de Comunicación Social, (71), 11701186. https://doi.org/10.4185/RLCS-2016-1140en

Smith, K. T. (2017). Hospital marketing and communications via social media. Services Marketing Quarterly, 38(3), 187-201. https://doi.org/1 0.1080/15332969.2017.1363518

Social Media Family (2019). V informe del uso de las redes sociales en españa. Social Media Family. Recuperado de https://thesocialmediafamily.com/informe-redes-sociales

Traish, A. M., Vance, J. C. y Morgentaler, A. (2017). Overselling hysteria: The role of the media and medical journals in promoting questionable risks - a case study of the testosterone controversy. EMBO Reports, 18(1), 11-17. https://doi.org/10.15252/embr.201643642

Triemstra, J. D., Poeppelman, R. S. y Arora, V. M. (2018). Correlations Between Hospitals' Social Media Presence and Reputation Score and Ranking: Cross-Sectional Analysis. Journal of Medical Internet Research, 2018, e289. https://doi.org/10.2196/jmir.9713

\section{CV}

Toni González Pacanowski. Es Profesor Titular en el Departamento de Comunicación y Psicología Social de la Universidad de Alicante, (UA) de España. Su investigación principal se centra en como los hospitales pueden integrar las redes sociales y las aplicaciones móviles en su funcionamiento interno para mejorar sus iniciativas de comunicación corporativa con los stakeholders internos y externos. Es autor de diversos libros relacionados con la comunicación médica y de artículos en los que se aborda la relación entre los públicos implicados en la interacción social que generan tanto los profesionales de la salud, la población y los medios de comunicación. Colabora como investigador en diversos proyectos oficiales del Ministerio de Educación del Gobierno de España.

Pablo Medina Aguerrebere. Es Doctor y Profesor Titular de la Facultad de Comunicación de la Cannadian University en Dubai. Experto en comunicación sanitaria, es uno de los principales investigadores internacionales en el ámbito hospitalario cen- 
trado en el management y la identidad corporativa. Ha definido diversos modelos y paradigmas para el análisis de procesos de la gestión comunicativa para centros sanitarios e instituciones relacionadas. Autor de diversos libros y numerosos artículos académicos su línea de investigación principal se orienta a la descripción de la actividad de los públicos relacionados con el flujo de información que se genera institucionalmente y que repercuten directamente en el beneficio de la salud.

Eva Medina. Es doctora por la Universidad de Alicante y licenciada en Derecho por la Universidad de París. Experta en comunicación de salud y su contexto legal internacional tiene amplia experiencia investigadora en Suiza y París, donde ha llevado a cabo investigaciones multicéntricas y en colaboración con diversos organismos europeos y entidades hospitalarias de primer nivel. Ha publicado numerosos artículos donde aborda el papel de la mujer en la relación médico-paciente, así como los públicos relacionados con los principales problemas de salud desde una perspectiva social y normativa. Actualmente colabora con diversos centros de investigación para el desarrollo de modelos de comunicación que optimice la gestión de los usuarios de la sanidad y atención social y sanitaria de la mujer.

Paula López Molina. Ha realizado diversos estudios sobre la comunicación publicitaria en el grupo de Investigación Publicitaria de la Universidad de Alicante y es autora de un proyecto sobre audiencias en internet en el que ha desarrollado sistemas de analítica para poder llevar a cabo la descripción de la actividad en redes sociales e internet de centros sanitarios. Actualmente su actividad se centra en el entorno profesional del márketing digital, área en la que ha cursado másters específicos por la UCAM y la Span Business School.

\section{осм Observatorio de Cibermedios}

https://observatoriocibermedios.upf.edu/

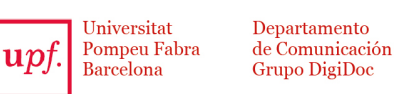

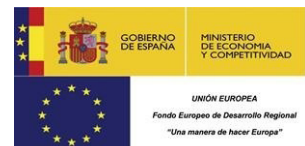

El Observatorio de Cibermedios es una producción del Grupo de Investigación en Documentación Digital y Comunicación Interactiva (DigiDoc) del Departamento de Comunicación de la Universitat Pompeu Fabra.

El Observatorio de Cibermedios (OCM) forma parte del proyecto del Plan Nacional "Narración interactiva y visibilidad digital en el documental interactivo y el periodismo estructurado". RTI2018-095714-B-C21 (MINECO/FEDER), Ministerio de Ciencia, Innovación y Universidades (España).

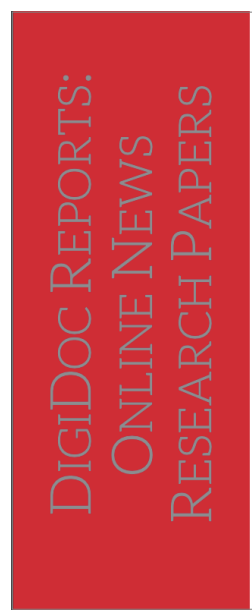

\section{Researching the news}

in the hybrid media system:

An expert panel report

Irene Costera Meijer, Richard Rogers,

Oscar Westlund \& Tamara Witschge

Javier Díaz-Noci \& Ana Serrano (eds.) 